\title{
Estimation of Panel Data Models with Binary Indicators when Treatment Effects are not Constant over Time
}

\author{
Audrey Laporte ${ }^{\mathrm{a},}$, Frank Windmeijer ${ }^{\mathrm{b}}$ \\ a Department of Health Policy, Management and Evaluation, University of Toronto, Toronto, Canada \\ ${ }^{\mathrm{b}}$ Cemmap, Institute for Fiscal Studies, London, UK
}

October 2004

\begin{abstract}
We show that two commonly employed estimation procedures to deal with correlated unobserved heterogeneity in panel data models, within-groups and first-differenced OLS, can lead to very different estimates of treatment effects when these are not constant over time and treatment is a state that only changes occasionally. It is therefore important to allow for flexible time varying treatment effects when estimating panel data models with binary indicator variables as is illustrated by an example of the effects of marital status on mental wellbeing.
\end{abstract}

Keywords: panel data, treatment effects

JEL Classification: C23, C51

\footnotetext{
- We would like to thank Stephen Bond and Brian S. Ferguson for helpful comments. Frank Windmeijer acknowledges financial support of the Leverhulme Trust. The usual disclaimer applies.

* Corresponding author. Department of Health Policy, Management and Evaluation, Faculty of Medicine, University of Toronto, McMurrich Bldg. $2^{\text {nd }}$ fl., 12 Queen's Park Cres. West, Toronto, Ontario, Canada M5S 1A8. E-mail: audrey.laporte@utoronto.ca; Fax: 416-978-7350; Phone: 416-946-7386
} 


\section{Introduction}

This paper considers the estimation of treatment effects in panel data models when these treatment effects are not constant over time. In particular, it analyses the differential effects on the commonly employed First-Differenced OLS (FDOLS) and Within-Groups (WG) estimation results when the treatment effects are misspecified to be instantaneous and constant, and where treatment is modelled with a binary indicator variable that only varies over time occasionally. A particular example that will be analysed is the effect of divorce on a measure of mental wellbeing, following Hauck and Rice (2004). Our estimation results indicate that divorce has an adverse effect on mental wellbeing that starts before the actual divorce takes place, peaks in the year of divorce and diminishes quite rapidly thereafter. A model that implies a constant instantaneous effect of divorce is therefore misspecified, leading to very different FDOLS and WG estimates.

The outline of the paper is as follows. Section 2 introduces the model and estimators. Section 3 explores the properties of the two estimators when the treatment effect varies over time. Section 4 proposes a straightforward solution to the misspecification and provides the empirical application that illustrates the technique. Section 5 concludes.

\section{Model Specification and Estimators}

Consider the estimation of a panel data model that contains a binary indicator or treatment variable as an explanatory variable. We write the equation to be estimated as

$$
Y_{i t}=\beta^{\prime} X_{i t}+\delta^{\prime} Z_{i}+\gamma D_{i t}+\alpha_{i}+v_{i t}
$$

where $i=1, \ldots, N$ indexes the individual in the panel and $t=1, \ldots, T$ indexes time. We are concerned with the usual micro-panel data, which means that the cross-section dimension $N$ is often large, but the time dimension $T$ small. $X_{i t}$ is a set of time-varying explanatory variables, $Z_{i}$ is a set of individual-specific, time-invariant variables and $D_{i t}$ is the $0-1$ binary variable for the status or treatment under consideration. Typically $D$ is a step variable, taking on a value of 0 in all periods prior to the change of status and 1 in all periods at and after the change. Implicit in this formulation is the assumption that the change in $D$ is accompanied immediately by a change in $Y$, and that the effect is full and permanent - there is no anticipation effect and no delayed 
response. Basically, as soon as the value of the binary variable switches from 0 to 1 , the intercept of the equation shifts by an amount $\gamma$.

Important is the presence of the unobserved individual heterogeneity term $\alpha_{i}$. This error component is often correlated with the explanatory variables which means that simple OLS on the pooled data results in biased and inconsistent estimates of $\beta, \gamma$ and $\delta$. The WG estimator remedies this problem by including the $N$ intercepts $\alpha_{i}$ as parameters to be estimated, which for the estimates for $\beta$ and $\gamma$ is equivalent to OLS in the model

$$
Y_{i t}-\bar{Y}_{i}=\beta^{\prime}\left(X_{i t}-\bar{X}_{i}\right)+\gamma\left(D_{i t}-\bar{D}_{i}\right)+\left(v_{i t}-\bar{v}_{i}\right),
$$

where $\bar{Y}_{i}, \bar{X}_{i}$ and $\bar{D}_{i}$ are the individual specific means, e.g. $\bar{Y}_{i}=\frac{1}{T} \sum_{t=1}^{T} Y_{i t}$. It is clear that WG will be consistent when $\left(X_{i t}-\bar{X}_{i}\right)$ and $\left(D_{i t}-\bar{D}_{i}\right)$ are uncorrelated with $\left(v_{i t}-\bar{v}_{i}\right)$, i.e. when $X_{i t}$ and $D_{i t}$ are strictly exogenous. Alternatively, the FDOLS estimator is OLS on the firstdifferenced model

$$
\Delta Y_{i t}=\beta^{\prime} \Delta X_{i t}+\gamma \Delta D_{i t}+\Delta v_{i t}
$$

which will result in consistent estimates if $\Delta X_{i t}$ and $\Delta D_{i t}$ are uncorrelated with $\Delta v_{i t} \cdot{ }^{1}$ As Wooldridge (2002) points out, WG is in general more efficient than FDOLS, but the latter is efficient when $v_{i t}$ is a random walk.

It is clear that for both the within groups and first differencing transformations all time invariant variables have vanished. The difference between the remaining $X_{i t}$ and $D_{i t}$ terms is that the $X_{i t}$ 's vary continuously while $D_{i t}$ might change its value only once for an individual, and indeed might not change at all - if $D_{i t}=1$ to indicate a divorced individual, anyone who does not become divorced during the observation period will have $D_{i t}=0 \forall t$, and someone who is divorced throughout the whole observation period will have $D_{i t}=1 \forall t . D_{i t}$ remains in the equation so long as it changes value in at least some of the individuals.

\footnotetext{
${ }^{1}$ The first-differenced model also often forms the basis for GMM estimation when regressors are endogenous. The findings as reported here for FDOLS will carry over to this GMM approach.
} 


\section{Sensitivity to Misspecification}

When (1) is the correct specification of the relation between $Y, X, Z$ and $D$, the WG and FDOLS estimators will result in similar estimates of $\gamma$. In this section we will evaluate the performance of the two estimators when there is a more flexible response to treatment that may not be constant over time. A general model specification that allows for this is

$$
Y_{i t}=\beta^{\prime} X_{i t}+\delta^{\prime} Z_{i}+\ldots+\gamma_{-2} P_{i,-2}+\gamma_{-1} P_{i,-1}+\gamma_{0} P_{i, 0}+\gamma_{1} P_{i, 1}+\gamma_{2} P_{i, 2}+\ldots+\alpha_{i}+v_{i t}
$$

where $\gamma_{j}\left(\gamma_{-j}\right)$ is the treatment effect $j$ periods after (before) the introduction of the treatment and the indicator variable $P_{i, j}\left(P_{i,-j}\right)$ is 1 in the $j$-th period after (before) the treatment, and 0 everywhere else. In the example of the effect of divorce on stress or mental health, it may well be that stress increases before the actual act of the divorce being completed. It is also likely in this case that stress levels just before and at the divorce date are actually higher than in the later periods after the divorce.

In the following we will ignore the presence of the other regressors and will investigate the behaviour of the WG and FDOLS estimators when estimating the model

$$
Y_{i t}=\gamma D_{i t}+\alpha_{i}+v_{i t}
$$

when the data are generated from

$$
Y_{i t}=\sum_{j=-l}^{r} \gamma_{j} P_{i, j}+\alpha_{i}+v_{i t}
$$

under various specifications of the $\gamma$ 's.

The WG and FD models are

$$
\begin{aligned}
Y_{i t}-\bar{Y}_{i} & =\gamma_{W G}\left(D_{i t}-\bar{D}_{i}\right)+\left(v_{i t}-\bar{v}_{i}\right) \\
\Delta Y_{i t} & =\gamma_{D} \Delta D_{i t}+\Delta v_{i t} .
\end{aligned}
$$

It is illustrative to consider the specific values of $\left(D_{i t}-\bar{D}_{i}\right)$ and $\Delta D_{i t}$. For an example where $T=7$ and an individual changes state in the $4^{\text {th }}$ period these are given by:

\begin{tabular}{|l|c|c|c|c|c|c|c|}
\hline Period & 1 & 2 & 3 & 4 & 5 & 6 & 7 \\
\hline$D$ & 0 & 0 & 0 & 1 & 1 & 1 & 1 \\
\hline$D_{i t}-\bar{D}_{i}$ & $-4 / 7$ & $-4 / 7$ & $-4 / 7$ & $3 / 7$ & $3 / 7$ & $3 / 7$ & $3 / 7$ \\
\hline$\Delta D_{i t}$ & - & 0 & 0 & 1 & 0 & 0 & 0 \\
\hline
\end{tabular}


It is clear that the model in differences is "short memory" and estimates the impact effect, whereas the WG transformation retains part of the structure of the state $D$.

The population regression parameter for the model in first differences, $\gamma_{D}$, is given by

$$
\gamma_{D}=\gamma_{0}-\gamma_{-1}
$$

Denote $t_{0}$ the period where $D$ switches from 0 to 1 . When all individuals in the sample switch state at the same time we get for the WG model

$$
\gamma_{W G}=\frac{1}{\left(T-t_{0}+1\right)} \sum_{j=0}^{T-t_{0}} \gamma_{j}-\frac{1}{t_{0}-1} \sum_{j=1}^{t_{0}-1} \gamma_{-j}=\bar{\gamma}_{0+}-\bar{\gamma}_{-}
$$

where $\bar{\gamma}_{0+}$ is the average of the $\gamma$ 's for the period $t_{0}$ and after, and $\bar{\gamma}_{-}$is the average of the $\gamma$ 's in the pre- $t_{0}$ period. If the proportion of people that switch state is the same for every $t_{0}=2, \ldots, T$, then the resulting "mixed" WG population parameter is given by

$$
\gamma_{W G}=\sum_{t_{0}=2}^{T}\left(w\left(t_{0}\right)\left(\bar{\gamma}_{0+}-\bar{\gamma}_{-}\right)\right)
$$

where the weights $w\left(t_{0}\right)$ are given by

$$
w\left(t_{0}\right)=\frac{\left(t_{0}-1\right)\left(T-t_{0}+1\right)}{\sum_{j=2}^{T}(j-1)(T-j+1)} .
$$

As is clear, $\gamma_{D}$ and $\gamma_{W G}$ can be very different when the treatment effects are not constant over time. We will calculate these population parameters for a selection of cases as presented in Table 1. In case I, the treatment effect is constant, but starts one period after treatment, i.e. there is no effect in period 0. In Case II the full treatment effect is obtained after 2 periods, with the effects in periods 0 and 1 being smaller and increasing. Case III is as Case II, but delayed with no effect in period 0. Case IV is also like Case II, but now there is a small anticipatory effect in period -1 . For Case $\mathrm{V}$ the long-term effect is again obtained after 2 periods, but now there are large initial, decreasing, effects in periods 0 and 1. Case VI is like Case V with an anticipatory effect in period -1 . Finally, Case VII only has an effect in periods 0 and 1 , with no long-run effect at all. For Cases I-VI the long-run treatment effect is 0.5 . 
Table 1. Various cases of treatment effect patterns

\begin{tabular}{|c|l|l|}
\hline & Treatment effects & Coefficient values \\
\hline I & Constant but delayed & $\gamma_{-j}=0, \gamma_{0}=0, \gamma_{1}=\gamma_{1+j}=0.5$ \\
\hline II & Increasing & $\gamma_{-j}=0, \gamma_{0}=0.25, \gamma_{1}=0.375, \gamma_{2}=\gamma_{2+j}=0.5$ \\
\hline III & Increasing but delayed & $\gamma_{-j}=0, \gamma_{0}=0, \gamma_{1}=0.25, \gamma_{2}=0.375, \gamma_{3}=\gamma_{3+j}=0.5$ \\
\hline IV & Anticipation, increasing & $\gamma_{-1-j}=0, \gamma_{-1}=0.10, \gamma_{0}=0.25, \gamma_{1}=0.375, \gamma_{2}=\gamma_{2+j}=0.5$ \\
\hline V & Declining, large initial & $\gamma_{-j}=0, \gamma_{0}=1.5, \gamma_{1}=1, \gamma_{2}=\gamma_{2+j}=0.5$ \\
\hline VI & $\begin{array}{l}\text { Declining, large initial, } \\
\text { anticipation }\end{array}$ & $\gamma_{-1-j}=0, \gamma_{-1}=0.75, \gamma_{0}=1.5, \gamma_{1}=1, \gamma_{2}=\gamma_{2+j}=0.5$ \\
\hline VII & Short run effect only & $\gamma_{-j}=0, \gamma_{0}=0.25, \gamma_{1}=0.375, \gamma_{2}=\gamma_{2+j}=0$ \\
\hline
\end{tabular}

Table 2 presents the population parameters $\gamma_{D}$ and $\gamma_{W G}$ for cases I-VII, again for $T=7$, where the differences between the two parameters are apparent for most cases. If one were interested in estimating the long-run effect of 0.5 in cases I-VI and 0 in Case 7, obviously WG is less biased for this long term effect than FDOLS, but it is furthermore clear that neither estimator is reliable.

Table 2. Population parameters $\gamma_{D}$ and $\gamma_{W G}$ for various cases of treatment effect patterns

\begin{tabular}{|c|c|c|c|c|c|c|c|c|}
\hline & $\gamma_{D}$ & \multicolumn{7}{|c|}{$\gamma_{W G}$} \\
\hline & & $t_{0}=2$ & $t_{0}=3$ & $t_{0}=4$ & $t_{0}=5$ & $t_{0}=6$ & $t_{0}=7$ & mixed \\
\hline I & 0 & 0.4167 & 0.4 & 0.375 & 0.3333 & 0.25 & 0 & 0.3125 \\
\hline II & 0.25 & 0.4375 & 0.425 & 0.4063 & 0.375 & 0.3125 & 0.25 & 0.3728 \\
\hline III & 0 & 0.3542 & 0.3250 & 0.2813 & 0.2083 & 0.125 & 0 & 0.2232 \\
\hline IV & 0.15 & 0.3375 & 0.3750 & 0.3729 & 0.35 & 0.2925 & 0.2333 & 0.3353 \\
\hline V & 1.5 & 0.75 & 0.8 & 0.875 & 1 & 1.25 & 1.5 & 1.0089 \\
\hline VI & 0.75 & 0 & 0.425 & 0.625 & 0.8125 & 1.1 & 1.375 & 0.7277 \\
\hline VII & 0.25 & 0.1042 & 0.125 & 0.1563 & 0.2083 & 0.3125 & 0.25 & 0.1942 \\
\hline
\end{tabular}

A test for the equivalence of the two parameters, $H_{0}: \gamma_{D}=\gamma_{W G}(\equiv \gamma)$ is easily obtained by estimating the extended model

$$
\left(\begin{array}{c}
Y_{i t}-Y_{i t-1} \\
Y_{i t}-\bar{Y}_{i}
\end{array}\right)=\left(\begin{array}{c}
D_{i t}-D_{i t-1} \\
D_{i t}-\bar{D}_{i}
\end{array}\right) \gamma+\left(\begin{array}{c}
0 \\
D_{i t}-\bar{D}_{i}
\end{array}\right) \phi+u_{i t}
$$

by OLS and testing $H_{0}: \phi=0$ by a standard t- or Wald test, using a robust variance estimate that allows for heteroskedasticity and (serial) correlation. Clearly, this test will not always have 
power to detect whether treatments effects are constant over time or not. For example, $\gamma_{D}$ and the mixed $\gamma_{W G}$ are quite close in Case VI, 0.75 and 0.73 respectively.

\section{Introducing Flexibility and Empirical Example}

The effects we have discussed are the result of specification errors. In particular, the use of a single step variable to reflect the treatment does not allow sufficient flexibility in the way the treatment effect can manifest itself in the dependent variable. It is of course quite straightforward to estimate the flexible specification (4), which will lead to consistent estimates of the $\gamma$ 's when estimated by WG or FDOLS. Below, we discuss estimation of this flexible model, while including $D$.

The $D$ variable often is, as noted above, a step variable, meaning that it assumes a value of 0 in all periods before the treatment occurs and 1 in the periods after. To add flexibility, we introduce a number of pulse variables, which assume the value 0 in all periods except one, and in that one period the value 1 . The equation is of the form:

$$
Y_{i t}=\beta^{\prime} X_{i t}+\delta^{\prime} Z_{i} \ldots+\gamma_{-2} P_{i,-2}+\gamma_{-1} P_{i,-1}+\gamma D_{i t}+\kappa_{0} P_{i, 0}+\kappa_{1} P_{i, 1}+\kappa_{2} P_{i, 2}+\ldots+\alpha_{i}+\varepsilon_{i t}
$$

where $\kappa_{j}=\gamma_{j}-\gamma$ for $j=0,1, \ldots$ and for identification one of the $P_{i, j}$ has to be omitted. The population regression parameters $\gamma_{D}$ and $\gamma_{W G}$ now have the correct value of 0.5 for Cases I-VI, and 0 for Case VII.

We illustrate the above discussion using data on GHQ scores from the British Household Panel Study (BHPS). GHQ stands for General Health Questionnaire and is a measure for mental wellbeing. There are 11 waves available, spanning the years 1991-2001, see Hauck and Rice (2004) for a description of the data and a more thorough analysis. ${ }^{2}$ We focus on the effect of divorce on the GHQ score in a sample of either married (or living as a couple) or divorced (separated) individuals. We only consider changes in status from being married to being divorced, and only one change can happen during the observation period. Table 3 reports the FDOLS and WG results for a simple model that includes time effects, the log of real household equivalised income and a quadratic age profile. There is a large and significant (short-run) effect of 2.3 for the model in first differences, whereas the within groups fixed effects estimator shows

\footnotetext{
${ }^{2}$ The GHQ score ranges from $0-36$, with the average in the pooled sample equal to 11.14 . A higher score is associated with a worse mental health state.
} 
a much smaller effect of divorce on the GHQ score of 0.6 , although still being significant at the $10 \%$ level. The test for equivalence of $\gamma_{D}$ and $\gamma_{W G}$, performed as described above, rejects the null, the difference $\gamma_{D}-\gamma_{W G}$ being 1.7 with an estimated robust standard error of 0.44 . Interestingly, equivalence of the other coefficients in the model is not rejected using the two different estimation procedures.

Table 3. Estimation results for GHQ model.

\begin{tabular}{|l|c|c|c|c|}
\hline & \multicolumn{2}{|c|}{ FDOLS } & WG \\
\hline & Coeff & Rob std err & Coeff & Rob std err \\
\hline Divorced & 2.2946 & .5289 & .5965 & .3156 \\
\hline \multicolumn{3}{|c|}{49153} & \multicolumn{2}{c|}{79649} \\
\hline \#obs & \multicolumn{2}{|c|}{7823} & \\
\hline $\mathrm{N}$ & $\begin{array}{l}\text { Other variables included in the regressions: year effects, age }{ }^{2}, \log \text { real household } \\
\text { equivalised income }\end{array}$ \\
\hline
\end{tabular}

Table 4 shows the estimation results of model (5) including $P_{i,-5}$ to $P_{i, 2} \cdot{ }^{3}$ The coefficient on divorced is now not significantly different from zero in both cases. There is a clear pattern of the effect of divorce on the GHQ score. There is a large effect at the time of divorce that is anticipated for quite a long period, but which declines rapidly after divorce. Figure 1 shows the pattern of the estimated $\gamma$ 's for both the WG and FDOLS estimators, for the model where the divorced indicator itself is excluded. The WG and FDOLS estimators show very similar results when allowing for the flexibility in the effects of divorce on GHQ scores.

Table 4. Estimation results for GHQ model including $P_{i,-5}$ to $P_{i, 2}$.

\begin{tabular}{|l|r|r|r|r|}
\hline & \multicolumn{2}{|c|}{ FDOLS } & \multicolumn{2}{|c|}{ WG } \\
\hline & Coeff & Rob std err & Coeff & \multicolumn{1}{c|}{ Rob std err } \\
\hline $\mathrm{P}_{-5}$ & .3950 & .5615 & .1393 & .4697 \\
\hline $\mathrm{P}_{-4}$ & .7531 & .6126 & .6090 & .5556 \\
\hline $\mathrm{P}_{-3}$ & 1.2031 & .5992 & 1.1201 & .5136 \\
\hline $\mathrm{P}_{-2}$ & 1.8793 & .6014 & 1.7613 & .5029 \\
\hline $\mathrm{P}_{-1}$ & 2.2371 & .6196 & 2.0827 & .5000 \\
\hline $\mathrm{P}_{0}$ & 4.5350 & .6706 & 4.2234 & .5544 \\
\hline
\end{tabular}

\footnotetext{
${ }^{3}$ Note that all the variables, including the step and pulse dummies have been transformed by first differencing or the within groups transformation.
} 


\begin{tabular}{|l|r|r|r|r|}
\hline $\mathrm{P}_{1}$ & .9866 & .5867 & 1.1467 & .4821 \\
\hline $\mathrm{P}_{2}$ & -.1697 & .5200 & .1967 & .4831 \\
\hline Divorced & -.0140 & .8034 & .1709 & .4741 \\
\hline \multicolumn{5}{|l|}{} \\
\hline \#obs & 49153 & 58645 & \\
\hline $\mathrm{N}$ & 7823 & 7919 & \\
\hline $\begin{array}{l}\text { Other variables included in the regressions: year effects, age, age }{ }^{2}, \log \text { real household } \\
\text { equivalised income }\end{array}$
\end{tabular}

\section{Conclusion}

We have shown that two commonly employed estimation procedures to deal with correlated unobserved heterogeneity in panel data models can lead to very different estimates of treatment effects when these are not constant over time. It is therefore important to allow for flexible time varying treatment effects when estimating panel data models with binary indicator variables as illustrated by our example of the effects of marital status on mental wellbeing.

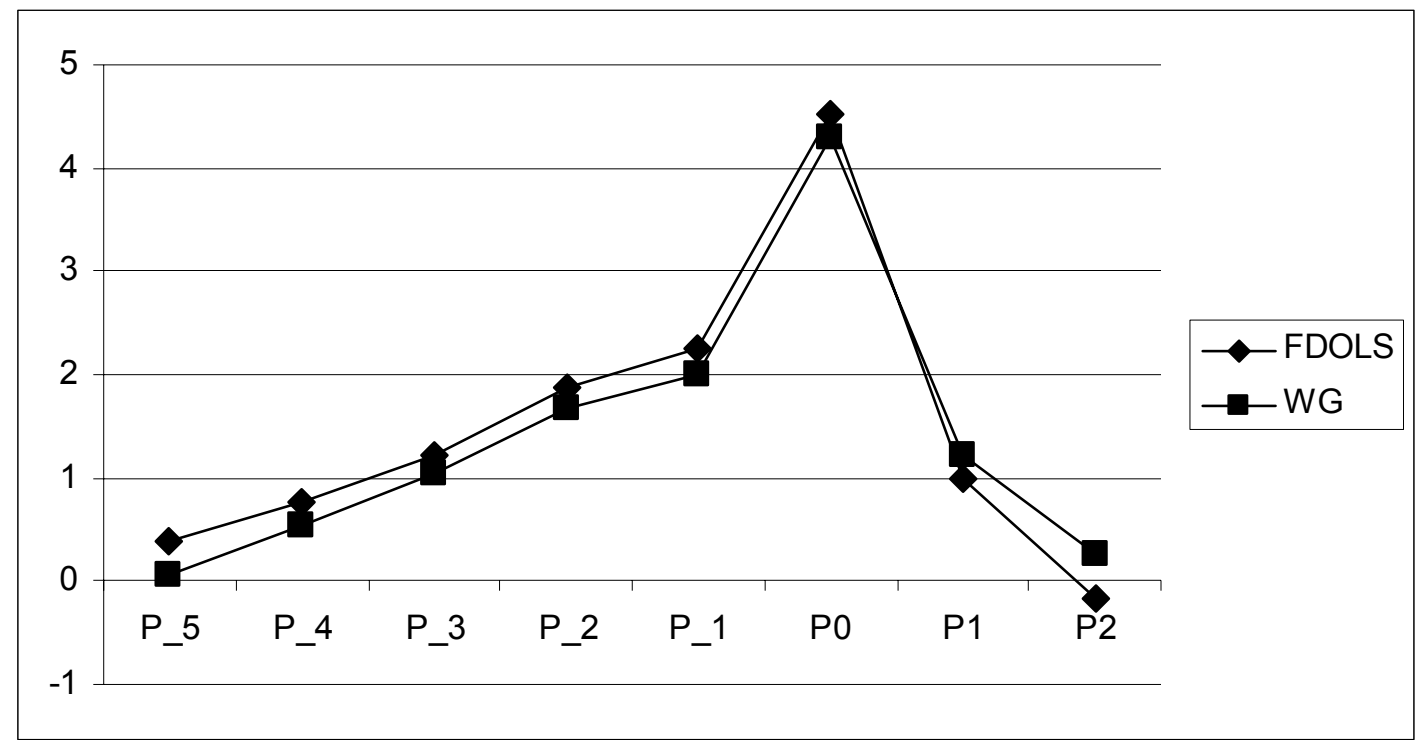

Figure 1. FDOLS and WG estimates of $\gamma_{j}$ for GHQ example 


\section{References}

Hauck, K. and N. Rice (2004), A Longitudinal Analysis of Mental Health Mobility in Britain, Health Economics 13, 981-1001.

Wooldridge, J.M. (2002), Econometric Analysis of Cross Section and Panel Data, The MIT Press. 